\title{
Quantitative Analysis of Oral Pathogenic Bacteria according to Smoking Using Real-Time PCR
}

\author{
Eun-Suk Jeon, Hyo-Jin Heo, and Hyo-Jin $\mathrm{Ko}^{\dagger}$ \\ Department of Dental Hygiene, Choonhae College of Health Sciences, Ulsan 44965, Korea
}

This study investigates the relationship between smoking and periodontal disease through quantitative analysis of intra-buccal oral pathogenic bacteria detected in smokers and aims to yield objective baseline data for applications in anti-smoking and dental health education programs. From April to May 2016, participants in an oral health management program within an intensive dental hygiene training course at Choonhae College of Health Sciences received an explanation of the study purposes and methods, after which male smokers aged 18 30 years agreed to participate voluntarily. Real-time polymerase chain reaction (PCR) analysis of oral pathogenic bacteria was performed after collecting gingival sulcus fluid samples from 67 smokers. The intra-buccal oral pathogenic bacteria distributions were analyzed based on the subjects' general characteristics, smoking behaviors, and oral care behaviors. The distribution results show that pathogens in the anterior teeth are affected (in this order) by age, toothbrush size, and smoking status; older people had fewer pathogens, those who used larger toothbrushes had more pathogens, and smokers had more pathogens, compared to non-smokers (adi $R^{2}=19.1$ ). In the posterior teeth, pathogens were influenced (in this order) by smoking status, smoking duration, and the number of tooth brushings per day; smokers had more pathogens than non-smokers, and those who brushed their teeth more often had fewer pathogens $\left({ }_{a d j} R^{2}=25.1\right)$. The overall pathogen distribution was affected only by smoking status: smokers generally had more pathogens, compared to non-smokers. Therefore, it is necessary to provide information about the risk of periodontal disease due to smoking during anti-smoking or dental health education sessions; particularly, the use of smaller toothbrushes for anterior teeth and the need for smokers in their early twenties to quit smoking for dental health should be highly emphasized.

Key Words: Oral pathogenic bacteria, Real-time polymerase chain reaction, Smokers

\section{Introduction}

Periodontal disease, a quintessential oral disease, involves the chronic infection of periodontal cells found in periodontal ligaments, the gums, cement, and alveolar bones, resulting in the destruction of cells that support the teeth. Periodontal diseases occur in response to various causes, such as microbes, dental prostheses, tooth loss, smoking, nutritional disorders, and genetic factors, and can be divided into inflammation of the gums, which involve infections of the gum only, and periodontitis, which involves the destruction of both the attached cells and alveolar bones ${ }^{1)}$. Microbes found in dental plaques are the core causes of such inflammation.
The oral cavity contains $300 \sim 500$ types of microbes, and in the normal gums, aerobic, inactive, gram-positive oral micrococcal species comprise the majority of these entities; however, the contraction of periodontal disease causes a shift to anaerobic, active gram-negative microbes ${ }^{2}$. Socransky et al. ${ }^{3)}$ asserted that periodontal diseases increase in the presence of anaerobic gram-negative microbes, and Greenstein and Polson ${ }^{4}$ asserted that the focal points of periodontal disease are an increase in the population of active microbes and reduction in micrococcal strains. In other words, changes in the composition of bacterial species and other microorganisms are associated with the occurrence of periodontal diseases. The key microbial species that cause periodontal diseases 
include Aggregatibacter actinomycetemcomitans, Porphyromonas gingivalis, and Prevotella intermedia. Of these, A. actinomycetemcomitans causes invasive periodontitis and facilitates periodontal cell destruction, $P$. gingivalis is associated with gum inflammation and alveolar bone destructions, and $P$. intermedia is known to facilitate hormone-related and complex periodontitis ${ }^{5)}$.

According to the 2016 statistics from the Organization for Economic Co-operation and Development (OECD), the percentage of daily smokers older than 15 years was $20.0 \%$ in the Korean population; this was higher than the OECD average of $18.6 \%{ }^{6}$. The 2012 Korean National Oral Health Survey ${ }^{7}$ reported a continuous increase in periodontal diseases among Korea adults, and found that smoking, dietary habits, stress, and the usage of oral hygiene items influenced oral health ${ }^{8,9)}$. Moreover, smoking is a known cause of general diseases such as lung cancer and cardiovascular diseases and is known to be related to epithelial attachment, alveolar bone loss, gum recession, and dental pocket formation ${ }^{8,10,11)}$. Barbour et al. ${ }^{12)}$ reported that smokers have a 2.5 to 6 -fold increased risk of periodontal diseases relative to non-smokers, while Won et al. ${ }^{13)}$ reported an increased prevalence of periodontal diseases among smokers, compared to nonsmokers. Reibel ${ }^{14)}$ also reported a higher prevalence of periodontal diseases among smokers than among non-smokers after comparing periodontal disease rates between the two groups, and also reported an increased likelihood of failure of dental treatments because of smoking.

To prevent periodontal diseases that destroy cells around the teeth and the alveolar bones, it is important to verify the composition of microorganisms within the oral cavity and quantify the results. Real-time polymerase chain reaction (PCR) is the most widely used technique for quantitatively observing the volume of microbes within the oral cavity, and involves the real-time quantification of acid levels throughout the PCR process to accurately quantify the substrates in each sample ${ }^{15)}$.

Research regarding the effects of smoking on periodontal diseases and associated correlations is ongoing ${ }^{8,10-14)}$; however, quantitative analyses of oral pathogenic bacteria according to oral cavity sections are lacking, particularly in terms of the associations of these characteristics with smoking behavior and oral health management.

Therefore, this study aimed to quantitatively analyze the intra-buccal oral pathogenic bacteria of smokers, identify the relationship between smoking and oral diseases, and provide objective baseline data that could be applied in anti-smoking and dental health education programs. Accordingly, this study sought to identify quantitative differences in oral pathogenic bacteria among different sections of the oral cavity based on smoking behavior and oral health management behavior with the intent to determine the influential factors.

\section{Materials and Methods}

\section{Subjects}

From April to May 2016, participants in an oral health management program within the intensive dental hygiene training course at C College, Ulsan Metropolitan City, received an explanation of the study purpose and method. A total of 67 male subjects between the ages of 18 and 30 years, including non-smokers, were enrolled after agreeing to participate voluntarily.

\section{Research method}

To ensure the ethical protection of the subjects, approval was obtained from the Institutional Review Board of Choonhae College of Health Sciences prior to the study (IRB approval no. CH-201604-12). The subjects received oral and written explanations of the study purpose and method, the commitment to confidentiality regarding study participation, the voluntary nature of participation, ability to opt in or out, and the potential advantages and disadvantages of participation; subsequently, agreement to participate was obtained from the subjects. A self-administered survey addressing sex, age, and general diseases was conducted, after which gingival sulcus fluid samples were collected from two anterior teeth and posterior teeth to compare quantitative differences in the oral pathogenic bacteria distributions by section. DNA was extracted from these samples, and multiplex real-time PCR was used to generate data. 


\section{1) Collection of gingival sulcus fluid}

To collect DNA from oral pathogenic bacteria, samples were collected from the subjects' oral cavities by inserting 4 sterilized no. 30 endodontic paper points (Sure Dent, Seongnam, Korea) for 10 seconds into the labial and lingual sections of the upper right central incisor and lower left central incisor among the anterior teeth, and by inserting 4 sterilized no. 90 endodontic paper points (Sure Dent) for 10 seconds in the buccal and lingual sections of the upper left first molar tooth and lower right first molar tooth. After removal, the paper points were frozen in $1.5-\mathrm{ml}$ micro tubes containing $1 \mu \mathrm{l}$ of phosphate-buffered saline (Gibco BRL, Grand Island, NY, USA) (two per each of the four teeth) and sent to the DNA lab where they were stored at $-20^{\circ} \mathrm{C}$ until use.

\section{2) DNA extraction of oral pathogenic bacteria and quantitative analysis}

An Exgene Clinic SV mini-kit (Biokorea, Cheongju, Korea) was used to extract DNA from oral microorganisms according to the manufacturer's protocol. For the quantitative analysis of oral pathogenic bacteria, a Cytoperio analysis system (Cytogen, Seoul, Korea) was used to expand the 16s rRNA and thus collect data of all microorganisms existing within the oral cavity.

Real-time PCR reactions were set so that the total volume was $20 \mu \mathrm{l}$; each reaction included $2 \mu \mathrm{l}$ of total extracted DNA, 10 pmol of each primer set, probe, and buffer solution, and 1 unit of Hot-start Taq DNA Polymerase (GeneAll, Seoul, Korea). The reactions were placed in 96-well plates and quantitatively analyzed using an ABI 7500 Fast Real-Time PCR System (Life Technologies Korea, Seoul, Korea). The real-time PCR conditions were as follows: denaturalization for 15 minutes at $95^{\circ} \mathrm{C}, 15$ seconds at $95^{\circ} \mathrm{C}$, and 45 cycles of 15 seconds at $55^{\circ} \mathrm{C}$ and 20 seconds at $72^{\circ} \mathrm{C}$. The cycle threshold $(\mathrm{Ct})$ analysis, which began in the exponential phase, used known DNA concentrations to measure the number of microbes in each sample according to the standard curve. In this study, the total volume of oral pathogenic bacteria is presented by section, and large numbers have been transformed into natural logs to simplify the comparison of differences.

\section{Statistical analysis}

The collected data were analyzed using IBM SPSS Statistics (ver. 21.0; IBM Co., Armonk, NY, USA) and the statistical significance level was at a $\mathrm{p}<0.05$. The subjects' general characteristics, smoking behavior, and oral health management behavior were analyzed using a frequency analysis, and a Student's t-test and one-way ANOVA were conducted to verify quantitative differences in oral pathogenic bacteria based on general characteristics, smoking behavior, and oral health management behavior. Moreover, a multiple regression analysis was conducted to identify the factors influencing quantitative differences in intra-buccal oral pathogenic bacteria.

\section{Results}

\section{Oral pathogenic bacteria and general characteristics}

In this study, $49.3 \%$ of the subjects were $18 \sim 22$ years, and $50.7 \%$ were $23 \sim 30$ years; furthermore, $67.2 \%$ were students, representing the largest subset of the population. Regarding residence, $50.7 \%, 35.8 \%$, and $13.4 \%$ of the subjects resided in Ulsan, Busan, and other regions, respectively. As noted, this study analyzed oral pathogenic bacteria by dental section, as well as the relationship of these pathogens with general characteristics. Among subjects aged $18 \sim 22$ years, $20.02 \pm 1.68$ and $20.21 \pm 1.66$ oral pathogenic bacteria were detected from the anterior and posterior teeth, with an overall number of $20.97 \pm 1.53$ pathogens; this was higher than the numbers detected in subjects aged $23 \sim 30$ years, in whom $18.64 \pm 1.35$ and $19.31 \pm 1.35$ were detected from the anterior and posterior teeth, with an overall number of $19.83 \pm 1.25$ pathogens ( $p$ $<0.05)$. However, no statistically significant differences were observed with respect to occupation or place of residence (Table 1).

\section{Oral pathogenic bacteria and smoking behavior}

The analysis of smoking behavior indicated that $55.2 \%$ of the subjects were non-smokers and $44.8 \%$ were smokers; of the former, the largest subset, $17.9 \%$, had smoked for 1 36 months. Furthermore, 29.9\% reported smoking fewer than 10 cigarettes per day, whereas $14.9 \%$ reported smoking more than 11 cigarettes per day. 
Table 1. Oral Pathogenic Bacteria according to General Characteristics

\begin{tabular}{lcccc}
\hline \multirow{2}{*}{ Characteristic } & $\mathrm{n}(\%)$ & \multicolumn{3}{c}{ Oral pathogenic bacteria } \\
\cline { 3 - 4 } Age $(\mathrm{y})$ & & Anterior & Posterior & Total microoganisms \\
$18 \sim 22$ & $33(49.3)$ & $20.02 \pm 1.68$ & $20.21 \pm 1.66$ & $20.97 \pm 1.53$ \\
$23 \sim 30$ & $34(50.7)$ & $18.64 \pm 1.35$ & $19.31 \pm 1.35$ & $19.83 \pm 1.25$ \\
p-value & & $<0.001^{* * *}$ & $0.017^{*}$ & $0.001^{* *}$ \\
Occupation & & & $19.72 \pm 1.20$ \\
Inoccupation & $9(13.4)$ & $18.71 \pm 1.38$ & $19.70 \pm 1.67$ & $20.13 \pm 1.10$ \\
Student & $45(67.2)$ & $19.32 \pm 1.74$ & $19.76 \pm 1.21$ & $20.35 \pm 1.61$ \\
Soldier & $4(6.0)$ & $18.91 \pm 1.60$ & $20.04 \pm 1.68$ & $20.13 \pm 1.33$ \\
Office workers & $9(13.4)$ & $20.09 \pm 1.48$ & 0.951 & $0.60 \pm 1.43$ \\
p-value & & 0.339 & & \\
Residence & & & $19.72 \pm 1.46$ & $20.22 \pm 1.42$ \\
Busan & $24(35.8)$ & $19.08 \pm 1.53$ & $19.73 \pm 1.61$ & $20.39 \pm 1.53$ \\
Ulsan & $34(50.7)$ & $19.30 \pm 1.75$ & $19.92 \pm 1.84$ & $20.81 \pm 1.70$ \\
Etc. & $9(13.4)$ & $20.00 \pm 1.67$ & 0.942 & 0.633 \\
p-value & & 0.375 & & \\
\hline
\end{tabular}

Values are presented as $\mathrm{n}(\%)$ or mean \pm standard deviation.

Bacteria levels were expressed as log scale of concentration.

${ }^{*} \mathrm{p}<0.05, * * \mathrm{p}<0.01,{ }^{* * *} \mathrm{p}<0.001$ statistically significant by t-test.

Table 2. Oral Pathogenic Bacteria Caused by Smoking Behavior

\begin{tabular}{|c|c|c|c|c|}
\hline \multirow{2}{*}{ Characteristic } & \multirow{2}{*}{$\mathrm{n}(\%)$} & \multicolumn{3}{|c|}{ Oral pathogenic bacteria } \\
\hline & & Anterior & Posterior & Total microoganisms \\
\hline \multicolumn{5}{|l|}{ Smoking } \\
\hline Non-smoking & $37(55.2)$ & $18.89 \pm 1.53$ & $19.30 \pm 1.46$ & $19.92 \pm 1.43$ \\
\hline Smoking & $30(44.8)$ & $19.84 \pm 1.70$ & $20.31 \pm 1.53$ & $20.94 \pm 1.41$ \\
\hline p-value & & $0.019 *$ & $0.008 * *$ & $0.005 * *$ \\
\hline \multicolumn{5}{|c|}{ Smoking period (mo) } \\
\hline Non-smoking & $37(55.2)$ & $18.89 \pm 1.53$ & $19.30 \pm 1.46^{\mathrm{a}}$ & $19.92 \pm 1.43^{\mathrm{a}}$ \\
\hline $1 \sim 36$ & $12(17.9)$ & $20.03 \pm 1.67$ & $20.89 \pm 1.18^{\mathrm{b}}$ & $21.35 \pm 1.16^{\mathrm{b}}$ \\
\hline $37 \sim 72$ & $9(13.4)$ & $19.91 \pm 1.97$ & $20.29 \pm 1.92^{\mathrm{a}, \mathrm{b}}$ & $20.88 \pm 1.88^{\mathrm{a}, \mathrm{b}}$ \\
\hline $73 \sim 120$ & $9(13.4)$ & $19.51 \pm 1.61$ & $19.57 \pm 1.32^{\mathrm{a}, \mathrm{b}}$ & $20.46 \pm 1.15^{\mathrm{a}, \mathrm{b}}$ \\
\hline p-value & & 0.114 & $0.011^{*}$ & $0.020^{*}$ \\
\hline \multicolumn{5}{|l|}{ 1-day smoking } \\
\hline Non-smoking & $37(55.2)$ & $18.89 \pm 1.53$ & $19.30 \pm 1.46^{\mathrm{a}}$ & $19.92 \pm 1.43^{\mathrm{a}}$ \\
\hline$\leq 10$ & $20(29.9)$ & $19.70 \pm 1.84$ & $20.45 \pm 1.52^{\mathrm{b}}$ & $20.93 \pm 1.50^{\mathrm{a}, \mathrm{b}}$ \\
\hline$\geq 11$ & $10(14.9)$ & $20.12 \pm 1.43$ & $20.04 \pm 1.59^{\mathrm{a}, \mathrm{b}}$ & $20.95 \pm 1.29^{\mathrm{b}}$ \\
\hline p-value & & 0.053 & $0.022 *$ & $0.020^{*}$ \\
\hline \multicolumn{5}{|l|}{ Family smoking } \\
\hline None & $32(47.8)$ & $19.06 \pm 1.67$ & $19.73 \pm 1.31$ & $20.29 \pm 1.34$ \\
\hline Father & $27(40.3)$ & $19.49 \pm 1.63$ & $19.71 \pm 1.75$ & $20.42 \pm 1.56$ \\
\hline Etc. & 8 (11.9) & $19.75 \pm 1.82$ & $20.02 \pm 2.03$ & $20.68 \pm 2.06$ \\
\hline p-value & & 0.462 & 0.879 & 0.816 \\
\hline
\end{tabular}

Values are presented as $\mathrm{n}(\%)$ or mean \pm standard deviation.

Bacteria levels were expressed as log scale of concentration.

${ }^{*} \mathrm{p}<0.05,{ }^{* *} \mathrm{p}<0.01$, statistically significant by t-test.

${ }^{*} \mathrm{p}<0.05$, statistically significant by one way ANOVA.

${ }^{\mathrm{a}, \mathrm{b}}$ Values are significantly different using the Scheffe method. 
Regarding family demographics, $40.3 \%$ had fathers who smoked. The analysis of oral pathogenic bacteria from teeth according to smoking behavior indicated that smokers contained anterior, posterior, and overall numbers of $19.84 \pm 1.70,20.31 \pm 1.53$, and $20.94 \pm 1.41$ pathogens, respectively, whereas non-smokers contained $18.89 \pm 1.53,19.30 \pm 1.46$, and $19.92 \pm 1.43$ pathogens respectively; in other words, smokers harbored higher numbers of oral pathogenic bacteria and microorganisms, compared to non-smokers $(p<0.05)$. The numbers of posterior and overall microorganisms differed significantly according to the length of smoking history and number of cigarettes per day; a post-hoc analysis revealed subjects who had smoked for $1 \sim 36$ months had $20.89 \pm 1.18$ and $21.35 \pm 1.16$ posterior and overall pathogens, respectively, compared to $19.30 \pm 1.46$ and $19.92 \pm 1.43$, respectively, for non-smokers. Smokers who consumed fewer than 10 cigarettes a day had $20.45 \pm 1.52$ and $20.93 \pm 1.50$ pathogens in the posterior and overall oral cavity, respectively, and both values were larger than the $19.30 \pm 1.46$ and $19.92 \pm 1.43$ pathogens identified respectively in non-smokers $(\mathrm{p}<0.05)$. However, there were no statistically significant differences associated with smoking by family members (Table 2 ).

\section{Oral pathogenic bacteria and oral health manage- ment behavior}

Regarding oral health management behavior, subjects who used larger toothbrushes harbored 19.62 \pm 1.66 anterior pathogens, a significantly larger number than the $18.74 \pm 1.54$ observed for those who used smaller toothbrushes $(\mathrm{p}<0.05)$. Subjects who brushed their teeth twice daily harbored $20.20 \pm 1.46$ anterior pathogens, compared to $18.80 \pm 1.31$ and $18.83 \pm 1.68$ for subjects who brushed once or thrice daily, respectively. Moreover, those who brushed twice daily had a higher number of overall

Table 3. Oral Pathogenic Bacteria according to Oral Health Behavior

\begin{tabular}{|c|c|c|c|c|}
\hline \multirow{2}{*}{ Characteristic } & \multirow{2}{*}{$\mathrm{n}(\%)$} & \multicolumn{3}{|c|}{ Oral pathogenic bacteria } \\
\hline & & Anterior & Posterior & Total microoganisms \\
\hline \multicolumn{5}{|l|}{ Toothbrush size } \\
\hline Small & $23(34.3)$ & $18.74 \pm 1.54$ & $19.46 \pm 1.45$ & $20.00 \pm 1.35$ \\
\hline Large & $44(65.7)$ & $19.62 \pm 1.66$ & $19.91 \pm 1.62$ & $20.59 \pm 1.55$ \\
\hline p-value & & $0.038^{*}$ & 0.273 & 0.126 \\
\hline \multicolumn{5}{|l|}{ No. of brushings per day } \\
\hline 1 & $11(16.4)$ & $18.80 \pm 1.31^{\mathrm{a}}$ & $20.16 \pm 1.16$ & $20.46 \pm 1.14^{\mathrm{a}, \mathrm{b}}$ \\
\hline 2 & $24(35.8)$ & $20.20 \pm 1.46^{\mathrm{b}}$ & $20.19 \pm 1.53$ & $20.95 \pm 1.46^{\mathrm{a}}$ \\
\hline 3 & $32(47.8)$ & $18.83 \pm 1.68^{\mathrm{a}}$ & $19.29 \pm 1.62$ & $19.91 \pm 1.52^{\mathrm{b}}$ \\
\hline p-value & & $0.004^{*}$ & 0.065 & $0.034^{*}$ \\
\hline \multicolumn{5}{|l|}{ Brushing time (min) } \\
\hline$\leq 2$ & $30(44.8)$ & $19.11 \pm 1.60$ & $19.57 \pm 1.44$ & $20.22 \pm 1.33$ \\
\hline$\geq 3$ & $37(55.2)$ & $19.49 \pm 1.72$ & $19.91 \pm 1.66$ & $20.51 \pm 1.63$ \\
\hline p-value & & 0.355 & 0.381 & 0.435 \\
\hline \multicolumn{5}{|c|}{ Toothbrush replacement interval (mo) } \\
\hline Bristle widening & $34(50.7)$ & $19.32 \pm 1.67$ & $19.69 \pm 1.59$ & $20.36 \pm 1.46$ \\
\hline 3 & $16(23.9)$ & $19.84 \pm 1.62$ & $19.93 \pm 1.60$ & $20.71 \pm 1.57$ \\
\hline$\geq 6$ & $17(25.4)$ & $18.82 \pm 1.63$ & $19.71 \pm 1.57$ & $20.13 \pm 1.55$ \\
\hline p-value & & 0.222 & 0.877 & 0.553 \\
\hline \multicolumn{5}{|l|}{ Oral hygiene devices } \\
\hline Do not use & $53(79.1)$ & $19.53 \pm 1.65$ & $19.86 \pm 1.57$ & $20.53 \pm 1.50$ \\
\hline Use & $14(20.9)$ & $18.52 \pm 1.53$ & $19.35 \pm 1.53$ & $19.83 \pm 1.41$ \\
\hline p-value & & $0.042 *$ & 0.283 & 0.121 \\
\hline
\end{tabular}

Values are presented as $\mathrm{n}(\%)$ or mean \pm standard deviation. Bacteria levels were expressed as log scale of concentration. ${ }^{*} \mathrm{p}<0.05$, statistically significant by one way ANOVA.

${ }^{\mathrm{a}, \mathrm{b}}$ Values are significantly different using the Scheffe method. 
microorganisms, $20.95 \pm 1.46$, compared to $19.91 \pm 1.52$ for those who brushed thrice daily $(\mathrm{p}<0.05)$. Subjects who did not use oral hygiene products harbored more anterior oral pathogenic bacteria when compared to those who did use such products $(19.53 \pm 1.65$ vs. $18.52 \pm 1.53, \mathrm{p}<0.05)$. However, no significant differences were observed with respect to the duration of brushing or duration of toothbrush use before replacement (Table 3).

\section{Factors influencing the distribution of oral pathogenic bacteria}

The ANOVA conducted to identify factors influencing the distribution of oral pathogenic bacteria revealed significant differences, and a stepwise multiple regression analysis of the following factors was conducted: age, smoker/non-smoker, smoking history, number of cigarettes a day, toothbrush size, number of tooth brushings per day, and usage of oral hygiene products. The anterior distribution of oral pathogenic bacteria was found to be influenced by (in this order) age, toothbrush size, and smoking; namely, older individuals harbored fewer oral pathogenic bacteria, and larger toothbrush users harbored 0.960 more oral pathogenic bacteria relative to smaller toothbrush users. Furthermore, smokers harbored 0.872 more oral pathogenic bacteria relative to non-smokers, and this factor had an explanatory power of $19.1 \%$. The posterior distribution of oral pathogenic bacteria was influenced by smoking, smoking history, and number of tooth brushings per day; namely, smokers harbored 2.125 more oral pathogenic bacteria relative to non-smokers. Furthermore, subject with longer smoking durations and those who more frequently brushed their teeth harbored fewer oral pathogenic bacteria, and these factors had an explanatory power of $25.1 \%$. The number of overall germs was only impacted by smoking; here, smokers harbored 1.561 more oral pathogenic bacteria, compared to non-smokers (Table 4).

\section{Discussion}

The Korean population has seen a continuous increase in the incidence of periodontal diseases among adults, and smoking, dietary habits, stress, and usage of oral hygiene items have been found to influence oral health ${ }^{8,9)}$. Moreover, Barbour et al. ${ }^{12)}$ reported that smokers have a 2.5 to 6-fold increased risk of periodontal disease, compared to non-smokers, and Won et al. ${ }^{13)}$ reported an increased prevalence of periodontal diseases among smokers, compared to non-smokers. Reibel ${ }^{14)}$ also reported a higher prevalence of periodontal diseases among

Table 4. Factors Affecting the Distribution of Oral Pathogenic Bacteria

\begin{tabular}{|c|c|c|c|c|c|c|}
\hline \multirow{2}{*}{ Characteristic } & \multicolumn{2}{|c|}{ Step I } & \multicolumn{2}{|c|}{ Step II } & \multicolumn{2}{|c|}{ Step III } \\
\hline & $\mathrm{B}$ & $\beta$ & $\mathrm{B}$ & $\beta$ & $\mathrm{B}$ & $\beta$ \\
\hline \multicolumn{7}{|l|}{ Anterior } \\
\hline Age & -0.230 & $-0.299 *$ & -0.238 & $-0.309 *$ & -0.212 & $-0.276^{*}$ \\
\hline Toothbrush size (large) & & & 0.924 & $0.399 *$ & 0.960 & $0.386^{*}$ \\
\hline Smoking (smoking) & & & & & 0.872 & $0.371^{*}$ \\
\hline${ }_{\text {adj }} R^{2}$ & \multicolumn{2}{|c|}{0.075} & \multicolumn{2}{|c|}{0.134} & \multicolumn{2}{|c|}{0.191} \\
\hline \multicolumn{7}{|l|}{ Posterior } \\
\hline Smoking (smoking) & 1.013 & $0.324 * *$ & 2.290 & $0.733 * * *$ & 2.125 & $0.680 * * *$ \\
\hline Smoking period & & & -0.023 & $-0.509 * *$ & -0.020 & $-0.438^{*}$ \\
\hline 1 day smoking & & & & & -0.392 & $-0.236^{*}$ \\
\hline${ }_{\text {adj }} R^{2}$ & \multicolumn{2}{|c|}{0.105} & \multicolumn{2}{|c|}{0.197} & \multicolumn{2}{|c|}{0.251} \\
\hline \multicolumn{7}{|l|}{ Total microoganisms } \\
\hline Smoking (smoking) & 1.561 & $0.270^{*}$ & & & & \\
\hline${ }_{\text {adj }} R^{2}$ & \multicolumn{2}{|c|}{0.058} & & & & \\
\hline
\end{tabular}

Smoking: non-smoking 0 , smoking 1 dummy variable.

Toothbrush size: small 0 , large 1 dummy variable.

$\mathrm{p}$-value determined from multiple linear reggression analysis.

${ }^{*} \mathrm{p}<0.05,{ }^{* *} \mathrm{p}<0.01, * * * \mathrm{p}<0.001$. 
smokers than among non-smokers, and observed that smoking increased the likelihood of dental treatment failure. Therefore, this study aimed to provide objective baseline data that could be applied in anti-smoking and dental health education programs, and thus quantitatively analyzed the intra-buccal oral pathogenic bacteria of smokers to identify the relationship between smoking and oral diseases. This analysis resulted in the following conclusions regarding the relationship between smoking and oral diseases.

Younger subjects (aged 18 22 years) harbored significantly more oral pathogenic bacteria, compared to older subjects $(23 \sim 30$ years $)(p<0.05)$. These results appear to be due to an increased interest in oral health and the practice of appropriate oral health management behaviors among subjects aged 23 years or older, which corresponds to entry into the workforce, compared to those in their early twenties.

Furthermore, smokers harbored more oral pathogenic bacteria and overall microorganisms relative to non-smokers in various areas of the oral cavity. These results are consistent with Haber et al. ${ }^{11)}$, who reported that $51 \%$ of patients with periodontal disease in the age group of 19 30 years were smokers, which suggests that smoking has a critically destructive influence on periodontal and oral diseases at fairly early ages (i.e., the twenties). Moreover, Haber et al. ${ }^{11)}$ also reported that among smokers, the loss of gum attachment was more pronounced in the anterior region and particularly in the lingual sections of the upper-front teeth, along with a tendency toward anterior gum recession. This study confirmed the presence of quantitative differences in oral pathogenic bacteria between smokers and non-smokers; although more oral pathogenic bacteria were detected in the posterior relative to the anterior section, there were no significant differences between the sections, which merits further research.

This study quantitatively analyzed oral pathogenic bacteria according to the length of smoking history; notably, those who had smoked for 1 36 months harbored more oral pathogenic bacteria, compared to non-smokers or smokers who had smoked for more than 37 months $(p<0.05)$. Haber et al. ${ }^{11)}$ asserted that the periodontal diseases begin at early ages (twenties and thirties), whereas disease progression occurs much more rapidly. However, Park et al. ${ }^{10)}$ researched the correlation between smoking and dental pocket formation and found no meaningful differences between smokers and non-smokers. This study confirmed the presence of more oral pathogenic bacteria in smokers in their twenties who had smoked for less than 36 months, compared to nonsmokers; the small decrease in oral pathogenic bacteria among smokers who had smoked for more than 37 months appeared to be due to the self-perception of issues such as oral odors and gum inflammation, and the consequent engagement in self-management.

In this study, smokers who consumed fewer than 10 cigarettes per day harbored more oral pathogenic bacteria in the posterior region, compared to non-smokers ( $\mathrm{p}$ $<0.05$ ). In a study by Lee and $\mathrm{Kim}^{16)}$ involving students of dentistry with relatively good oral health, the authors classified the subjects by a smoking history of less than 5 years $(38.3 \%)$ and $5 \sim 10$ years $(38.3 \%)$; the majority had smoked for less than 10 years, and almost $90 \%$ smoked fewer than 20 cigarettes a day. Similarly, in this study, $40 \%$ of the smokers had smoked for less than 36 months, and the majority had not smoked for more than 10 years; $67 \%$ of smokers smoked fewer than 10 cigarettes, and only $33 \%$ smoked more than 10 cigarettes a day. Calsina et al. ${ }^{17)}$ emphasized the presence of a dose-effect relationship between smoking and the prevalence of periodontal diseases and asserted that the influence of smoking on periodontal cells becomes more pronounced after 10 years, independent of factors such as age, sex, and the plaque index. Tomar and $\mathrm{Asma}^{18)}$ asserted that the daily number of cigarettes is an important risk determinant; specifically, light smokers experience a twofold increase in the risk of periodontitis, and this risk increases to six-fold among heavy smokers (more than 30 cigarettes per day). The authors also indicated that the prevalence of periodontitis decreased with smoking cessation. However, no significant differences were observed between smokers who smoked more than 10 and fewer than 10 cigarettes; this observation, which appears to be due to the smaller sample size of this study, merits further research. 
The analysis of the relationship between oral health management behavior and oral pathogenic bacteria suggests the presence of more anterior oral pathogenic bacteria in subjects who used larger toothbrushes, brushed their teeth twice daily, and did not use oral hygiene products $(\mathrm{p}<0.05)$. Won et al. ${ }^{13}$ reviewed the relationship between periodontal diseases and oral health behaviors and observed periodontal diseases in $36.7 \%$ of subjects who brushed less than once daily, $24.7 \%$ of subjects who did not use dental floss, and $23.5 \%$ of subjects who did not use interdental toothbrushes. This study confirmed a higher number of anterior oral pathogenic bacteria when larger toothbrushes were used; specifically, larger toothbrush heads led to a failure of contact between the actual brush and the sides of anterior teeth, leading to a higher prevalence of oral diseases. However, this study also indicated a higher number of oral pathogenic bacteria among subjects who brushed their teeth twice daily, compared to once or thrice daily, and this finding appears to merit future research on various factors such as the brushing methods and duration and the type of toothpaste used. Moreover, as this study indicated that the usage of oral hygiene products reduced the number of anterior oral pathogenic bacteria, the usage of products such as dental floss and interdental toothbrushes may reduce the risk of periodontal and oral diseases.

This cross-sectional study evaluated a convenience sample of male smokers and non-smokers in their twenties, and therefore the generalizability of the results is limited. Moreover, further research is required, as this study failed to clearly present quantitative differences in the types of germs that cause periodontal diseases. Despite such limitations, this study quantitatively analyzed the intra-buccal oral pathogenic bacteria harbored by smokers, identified the relationship between smoking and oral diseases, and provided objective baseline data that can be applied in anti-smoking education and dental health education programs. Furthermore, this study is significant because it has identified quantitative differences in oral pathogenic bacteria among different sections of the oral cavity in association with smoking behavior and oral health management behavior.

Oral diseases, including periodontal diseases, appear to be influenced by age, smoking status, smoking history, number of cigarettes per day, toothbrush size, and the use of oral hygiene products. Moreover, it is important to broadly educate the population about the risk of oral diseases from smoking through anti-smoking and oral health education programs. It is also important to emphasize the use of an appropriate toothbrush size and to further emphasize the need for smoking cessation to ensure healthy oral cavity maintenance among smokers in their twenties. Because the appropriate use of oral hygiene products is related to periodontal health and the prevention of oral diseases, education about the need for such products and usage methods must be consistently provided.

\section{Acknowledgements}

This research was supported by Choonhae College of Health Sciences in 2016.

\section{References}

1. Newman MG, Takei HH, Klokkevold PR, Carranza FA: Carranza's clinical periodontology. 11th ed. Saunders Elsevier, St. Louis, pp.45-75, 2012.

2. Harris NO, Garcia-Godoy F: Primary preventive dentistry. 6th ed. Pearson Education, Upper Saddle River, pp.73-92, 2004.

3. Socransky SS, Haffajee AD, Cugini MA, Smith C, Kent RL Jr: Microbial complexes in subgingival plaque. J Clin Periodontol 25: 134-144, 1998. https://doi.org/10.1111/j.1600-051X.1998.tb02419.x

4. Greenstein G, Polson A: Microscopic monitoring of pathogens associated with periodontal diseases. A review. J Periodontol 56: 740-747, 1985.

https://doi.org/10.1902/jop.1985.56.12.740

5. Loomer PM: Microbiological diagnostic testing in the treatment of periodontal diseases. Periodontol 2000 34: 49-56, 2004. https://doi.org/10.1046/j.0906-6713.2002.003424.x

6. OECD: Health statistics 2016. Ministry of Health and Welfare, Seoul, pp.50-53, 2016.

7. Ministry of Health and Welfare, Disease Control Division: Korean national oral health survey report 2012. Ministry of 
Health and Welfare, Seoul, pp.1-694, 2012.

8. Shizukuishi S: Smoking and periodontal disease. Clin Calcium 17: 226-232, 2007.

9. Ronis DL, Lang WP, Farghaly MM, Passow E: Tooth brushing, flossing, and preventive dental visits by Detroitarea residents in relation to demographic and socioeconomic factors. J Public Health Dent 53: 138-145, 1993.

https://doi.org/10.1111/j.1752-7325.1993.tb02692.x

10. Park JH, Kim YN, Yoo JH, Kim MY, Kim BI, Kwon HK: Relationship between smoking and periodontal pocket formation in Korean adults. J Korean Acad Oral Health 29: 293-301, 2005.

11. Haber J, Wattles J, Crowley M, Mandell R, Joshipura K, Kent $\mathrm{RL}$ : Evidence for cigarette smoking as a major risk factor for periodontitis. J Periodontol 64: 16-23, 1993. https://doi.org/10.1902/jop.1993.64.1.16

12. Barbour SE, Nakashima K, Zhang JB, et al.: Tobacco and smoking: environmental factors that modify the host response (immune system) and have an impact on periodontal health. Crit Rev Oral Biol Med 8: 437-460, 1997. https://doi.org/10.1177/10454411970080040501

13. Won YS, Choi $\mathrm{CH}$, Oh $\mathrm{HN}$ : Risk factors of periodontal disease in Korean adults. J Korean Acad Oral Health 38: 176-183, 2014. https://doi.org/10.11149/jkaoh.2014.38.3.176

14. Reibel J: Tobacco and oral diseases. Update on the evidence, with recommendations. Med Princ Pract 12 Suppl 1: 22-32, 2003. https://doi.org/10.1159/000069845

15. Kim SM, Yang KH, Choi NK, Kang MS, Oh JS: Quantitative detection of periodontopathic bacteria using real-time PCR. J Korean Acad Pediatr Dent 35: 494-503, 2008.

16. Lee HS, Kim ME: Effects of smoking on oral health: preliminary evaluation for a long-term study of a group with good oral hygiene. J Oral Med Pain 36: 225-234, 2011.

17. Calsina G, Ramón JM, Echeverría JJ: Effects of smoking on periodontal tissues. J Clin Periodontol 29: 771-776, 2002. https://doi.org/10.1034/j.1600-051X.2002.290815.x

18. Tomar SL, Asma S: Smoking-attributable periodontitis in the United States: findings from NHANES III. National Health and Nutrition Examination Survey. J Periodontol 71: 743-751, 2000. https://doi.org/10.1902/jop.2000.71.5.743 